\title{
AVALIAÇÃO DO CONTEÚDO DE UM CURSO DE ESPECIALIZAÇÃO OFERTADO AOS PROFISSIONAIS DE SAÚDE NA MODALIDADE EAD
}

\author{
Camila Santos de Castro e Lima ${ }^{1}$, Ana Emília Figueiredo de Oliveira ${ }^{2}$, \\ Mizraim Nunes Mesquita ${ }^{3}$, Deborah de Castro e Lima Baesse ${ }^{4}$, Fernanda Ferreira Lopes ${ }^{5}$, \\ Elza Ferreira Bernardes ${ }^{6}$
}

Resumo: A Educação a Distância (EaD), fruto da evolução e popularização dos recursos tecnológicos, é uma metodologia de ensino que permite aos alunos a interação com professores, tutores e instituição em tempo e local físico diferentes, através de algum tipo de tecnologia. Este trabalho é o resultado de um estudo realizado a partir de uma amostragem de natureza quantitativa e da aplicação de questionários, que investigou a avaliação e satisfação dos alunos quanto ao conteúdo de um curso de especialização ofertado na modalidade $\mathrm{EaD}$ aos profissionais da saúde vinculados ao Sistema Único de Saúde (SUS). Assim, foi possível conhecer o nível de satisfação do aluno em relação ao conteúdo, à estrutura e à aprendizagem obtida no curso em questão, favorecendo a compreensão dos aspectos citados e indicando tendências que possibilitarão a produção de objetos de aprendizagem mais eficazes.

Palavras-chave: Educação a Distância. Educação permanente. Profissional de saúde. Objetos de aprendizagem.

1 Graduanda em Design pela Universidade Federal do Maranhão - UFMA.

2 Pós-doutora e Professora Associada da Universidade Federal do Maranhão.

3 Graduanda em Letras pela Universidade Federal do Maranhão - UFMA. Pesquisadora vinculada ao $\mathrm{PIBIC/CNPq}$.

4 Doutoranda em Ciências Médicas pela Universidade do Estado do Rio de Janeiro, UERJ, Brasil. Professora da Universidade Federal do Maranhão.

5 Doutora em Patologia Oral pela Universidade Federal do Rio Grande do Norte, UFRN, Brasil. Professora adjunto da Universidade Federal do Maranhão.

6 Doutoranda do Programa de Pós-Graduação em Ciências Médicas (UERJ). 


\title{
CONTENT ASSESSMENT OF A SPECIALIZATION COURSE OFFERED TO HEALTH PROFESSIONALS THROUGH DISTANCE LEARNING
}

\begin{abstract}
Distance education modality originated from the evolution and popularization of technological resources is a learning method that allows students to interact with teachers, tutors and institution on a different time and physical place through some kind of technology. This research is the result of a study from a quantitative sampling, through the use of questionnaires, which investigated the student's assessment and satisfaction regarding the contents of a specialization course offered in distance education modality to health professionals linked to Brazilian National Health System (SUS). Therefore, it was possible to know the student's satisfaction level regarding the content, structure and obtained knowledge in the determined course, enabling the understanding from the referred aspects and indicating trends so that more effective learning objects can be produced.
\end{abstract}

Keywords: Distance education modality. Permanent Education. Health professional. Learning objects.

\section{INTRODUÇÃO}

O desenvolvimento das tecnologias influencia diretamente diversos setores da sociedade, dentre eles a educação. Como resultado desse avanço tecnológico, observase, nos ultimos tempos, um forte investimento na modalidade Educação a Distância $(\mathrm{EaD})$.

A evolução da EaD está ligada diretamente ao desenvolvimento tecnológico, aos avanços da informática e aos meios de comunicação em massa (NOGUEIRA, 1996). Um dos fatores que possibilitaram o desenvolvimento dessa metodologia foi o uso da Internet, que está presente de forma bastante determinante em diversos campos sociais, como o empresarial, o social e o cultural, não sendo diferente no campo educacional permitindo a exploração de diferentes metodologias e concepções pedagógicas que visam melhorar a eficiência em cursos tradicionais presenciais e em cursos na $\mathrm{EaD}$ (SCHRÖEDER, 2009).

O termo Educação a Distância normalmente refere-se ao processo de ensinoaprendizagem mediado pelas Tecnologias de Informação e Comunicação (TICs ${ }^{7}$ ), tais como os computadores conectados à internet. Nesta modalidade de ensino, os agentes envolvidos - professores, conteudistas, tutores e alunos - não estão presentes ao mesmo tempo e no mesmo espaço físico, e a mediação didáticopedagógica é realizada por meio de TICs e Artefatos Digitais de Aprendizagem (MEC, 2007 apud PUERTA; AMARAL, 2012).

7 Tecnologias da Informação e Comunicação - TICs são recursos tecnológicos integrados entre si, mediadores do processo informacional. Disponível em: <http://www.infoescola.com/informatica/ tecnologia-da-informacao-e-comunicacao/>. 
Essa modalidade de ensino tem como alguns dos princípios norteadores: a flexibilidade, permitindo mudanças durante o processo; a contextualização, onde cada curso é desenvolvido de acordo com as necessidades de cada região; a diversificação, através do desenvolvimento de atividades e materiais que permitam diversas formas de aprendizagem; a abertura, que permite que o aluno organize seu tempo e espaço de acordo com as suas necessidades (LEITE, 1998 apud GARCIA et al., 2015).

Por isso, a EaD exige técnicas especiais para a criação, instrução, comunicação por meio de diversas tecnologias, organização e administração (MOORE; KEARSLEY, 2007). Para que o aluno tenha acesso ao material de ensino e interaja com os outros integrantes do processo ensino-aprendizagem (professores, tutores, alunos e instituição), a EaD faz uso dos Ambientes Virtuais de Aprendizagem (AVAs), sistemas computacionais disponibilizados na internet, destinados ao suporte de atividades educacionais mediadas pelas TICs. Estes ambientes integram diversas mídias, linguagens e recursos, apresentando informações de maneira organizada, promovendo interações entre pessoas e objetos de aprendizagem, de acordo com o tempo, ritmo e espaço de cada participante (ALMEIDA, 2003 apud BONICI; ARAÚJO JÚNIOR, 2011).

Os recursos desenvolvidos para a $\mathrm{EaD}$, definidos aqui como objetos de aprendizagem, são materiais educacionais que servem para apoiar o processo de ensinoaprendizagem e permitem aperfeiçoar o material didático, através do uso de recursos multimídia e da interatividade aluno-curso, aluno-tutor e aluno-aluno, dentro do AVA. Incluem-se cursos, livros, vídeos, coleções da biblioteca, jogos, revistas científicas e artigos, simulações e avaliações e o software aberto que apoia a sua criação, hospedagem e divulgação, entre outros (MAYADAS; BOURNE; BACSICH, 2009).

Para a modalidade $\mathrm{EaD}$, os requisitos básicos costumam ser computadores pessoais convencionais, com conexão à Internet, a partir dos quais professores e alunos participam das aulas utilizando um software para gerenciamento do curso, que possibilitam tanto a interação assíncrona quanto a síncrona, como por exemplo discussões simultâneas (MAYADAS; BOURNE; BACSICH, 2009).

A principal razão e o foco da educação à distância é o considerável número de indivíduos que buscam por aperfeiçoamento, geralmente com certificação, e que, por diversos motivos, não podem comparecer a um curso presencial. Normalmente, são adultos trabalhadores que não podem integrar iniciativas educacionais presenciais por restrições de tempo relacionadas a família, trabalho, etc. Por tais motivos, a $\mathrm{EaD}$ vem sendo cada vez mais procurada e utilizada. De acordo com o levantamento realizado pela Associação Brasileira de Educação a Distância - ABED, no Censo EaD.br, realizado em 2013, houve um aumento no números de matrícula em cursos nesta modalidade em torno de 32\%, com um total de cursos EaD informados de 15.733 (ABED, 2014).

Os profissionais que atuam na área da saúde tem sido constantemente alvo de iniciativas no âmbito da Educação a Distância. As intensas jornadas de trabalho e a escassez de tempo costumam ser características frequentes na vida destes indivíduos, 
o que muitas vezes os impedem de integrar a Educação Permanente em Saúde (EPS), importante mecanismo de formação continuada e aperfeiçoamento de práticas na área.

A fim de atender a esta demanda por EPS, o governo brasileiro, através do Ministério da Saúde (MS), criou em 2010 o Sistema Universidade Aberta do SUS (UNA-SUS), que se responsabiliza desde então pela capacitação e educação permanente dos trabalhadores do SUS, fazendo uso da EaD. Desta forma, facilitou-se o acesso dos profissionais da saúde à EPS, já que as tecnologias da informação e comunicação possibilitam a ampliação do alcance deste tipo de atividade educativa (OLIVEIRA et al., 2014).

O Sistema UNA-SUS opera em parceria com instituições de ensino superior, contando atualmente com 36 unidades espalhadas pelo Brasil. Entre estas instituições está a UNA-SUS/UFMA que, com o suporte da Universidade Federal do Maranhão (UFMA), atua ofertando formação continuada especialmente para os profissionais que trabalham nessa região, dentro do SUS.

A UNA-SUS/UFMA é a instituição com a maior oferta de cursos dentro do Sistema UNA-SUS. Por essa razão, faz-se necessário avaliar a satisfação dos alunos e a eficácia dos recursos e metodologias utilizadas, para que se possa identificar possíveis pontos a serem aprimorados e estratégias bem sucedidas a serem reproduzidas por outras instituições que trabalham com EaD. Nesse sentido, o presente trabalho pretende investigar a satisfação do aluno em relação ao conteúdo e à sua aprendizagem em um curso de especialização ofertado pela UNA-SUS/UFMA aos profissionais da Atenção Básica do SUS.

\section{METODOLOGIA}

Esta pesquisa foi aprovada pelo Comitê de Ética em Pesquisa na Universidade Federal do Maranhão, sob o No 715.841. Para a condução da mesma, participaram da amostra os alunos regularmente matriculados no Curso de Especialização em Saúde da Família, cujo público-alvo era constituído dos participantes do Programa de Valorização dos Profissionais da Atenção Básica (PROVAB). O curso foi ofertado na modalidade Educação a Distância pela Universidade Aberta do Sistema Único de Saúde da Universidade Federal do Maranhão (UNA-SUS/UFMA). Foram excluídos da amostra os estudantes que desistiram antes do início do curso ou que não acessaram o AVA nenhuma vez, de modo que um total de 118 alunos participaram da pesquisa.

Para a coleta de dados utilizou-se um questionário, aplicado de forma presencial nas datas 06 de dezembro de 2014 e 06 de fevereiro de 2015, com respostas categóricas ordinais seguindo uma escala likert (discordo totalmente; discordo; concordo mais ou menos; concordo; concordo totalmente), com foco nos seguintes aspectos: avaliação de conteúdo, estrutura do curso, satisfação dos pós-graduandos, e avaliação do resultado de aprendizagem. 
Os dados foram analisados utilizando os recursos do software SPSS Statistical Software (versão 17.0, IBM SPSS Inc., Chicago, IL, USA). Inicialmente, foi realizada a estatística descritiva das dimensões de avaliação utilizando frequências absolutas e relativas. Média e desvio-padrão foram utilizadas para quantificar o uso semanal do livro on-line. O coeficiente de Spearman (Rs) foi utilizado para estimar a correlação entre as dimensões de avaliação (conteúdo, estrutura, satisfação e aprendizagem). Para essa análise os escores categóricos ordinais foram calculados e somados para cada uma das dimensões de avaliação. O nível de significância adotado foi de 5\%.

\section{RESULTADOS}

A distribuição dos escores de avaliação do conteúdo do curso está expressa na Tabela 1. Das variáveis que apresentaram maior percentual de avaliação positiva (concordo/concordo totalmente), o questionamento se conteúdo era claro e fácil de entender apresentou $92,4 \%$ de avaliações positivas, seguido pelas questões sobre se o conteúdo do curso corresponde aos objetivos definidos pelo curso $(91,5 \%)$ e se os materiais e recursos melhoraram a aprendizagem do curso (89,7\%). Vale mencionar que para todas a questões deste bloco, as avaliações positivas foram maiores de $70 \%$.

Tabela 1 - Avaliação do conteúdo de um curso de especialização na modalidade EaD por pós-graduandos utilizando uma escala de likert

\begin{tabular}{|c|c|c|}
\hline Avaliação de conteúdo & $\mathbf{n}$ & $(\%)$ \\
\hline \multicolumn{3}{|c|}{ O conteúdo do curso corresponde aos objetivos definidos pelo curso } \\
\hline Discordo totalmente & 2 & $(1,7)$ \\
\hline Discordo & 0 & $(0)$ \\
\hline Concordo mais ou menos & 8 & $(6,8)$ \\
\hline Concordo & 75 & $(63,6)$ \\
\hline Concordo totalmente & 33 & $(27,9)$ \\
\hline \multicolumn{3}{|c|}{ Os materiais/recursos do curso melhoraram a aprendizagem do assunto } \\
\hline Discordo totalmente & 0 & $(0)$ \\
\hline Discordo & 2 & $(1,7)$ \\
\hline Concordo mais ou menos & 10 & $(8,6)$ \\
\hline Concordo & 74 & $(63,2)$ \\
\hline Concordo totalmente & 31 & $(26,5)$ \\
\hline \multicolumn{3}{|c|}{ O conteúdo abordou minhas necessidades de aprendizagem } \\
\hline Discordo totalmente & 1 & $(0,9)$ \\
\hline Discordo & 4 & $(3,4)$ \\
\hline Concordo mais ou menos & 26 & $(22,0)$ \\
\hline Concordo & 61 & $(51,7)$ \\
\hline Concordo totalmente & 26 & $(22,0)$ \\
\hline
\end{tabular}




\begin{tabular}{lccc}
\hline & Avaliação de conteúdo & n & (\%) \\
\hline O conteúdo era claro e fácil de entender & & \\
\hline Discordo totalmente & 0 & $(0)$ \\
Discordo & 0 & $(0)$ \\
Concordo mais ou menos & 9 & $(7,6)$ \\
Concordo & 59 & $(50,0)$ \\
Concordo totalmente & 50 & $(42,4)$ \\
\hline O conteúdo era aplicável à minha prática & & \\
\hline Discordo totalmente & 1 & $(0,9)$ \\
Discordo & 4 & $(3,4)$ \\
Concordo mais ou menos & 28 & $(23,9)$ \\
Concordo & 55 & $(47,0)$ \\
Concordo totalmente & 29 & $(24,8)$ \\
\hline O curso será de grande valia para o desenvolvimento do meu trabalho & & \\
\hline Discordo totalmente & 2 & $(1,7)$ \\
Discordo & 2 & $(1,7)$ \\
Concordo mais ou menos & 17 & $(14,4)$ \\
Concordo & 57 & $(48,3)$ \\
Concordo totalmente & 40 & $(33,9)$ \\
\hline
\end{tabular}

Fonte: dos autores.

A Tabela 2 apresenta a distribuição das variáveis de avaliação da estrutura do curso. Todos os questionamentos apresentaram avaliações positivas com percentuais maiores que 70\%. Destacaram-se as questões: o curso online foi fácil de usar; as páginas foram claramente definidas; e o material do curso foi organizado de forma lógica e compreensível - que apresentaram, respectivamente, percentuais positivos de 88,8\%, $88 \%$ e $88 \%$.

Tabela 2 - Avaliação da estrutura de um curso de especialização na modalidade EaD por pós-graduandos utilizando uma escala de likert

\begin{tabular}{lcc}
\hline \multicolumn{1}{c}{ Avaliação da estrutura do curso } & n & (\%) \\
\hline O site do curso tinha facilidade de uso & & \\
\hline Discordo totalmente & 1 & $(0,9)$ \\
Discordo & 5 & $(4,3)$ \\
Concordo mais ou menos & 21 & $(17,9)$ \\
Concordo & 49 & $(41,9)$ \\
Concordo totalmente & 41 & $(35,0)$ \\
\hline Os objetivos e procedimentos do curso foram claramente comunicados & & \\
\hline Discordo totalmente & 1 & $(0,9)$ \\
Discordo & 3 & $(2,6)$
\end{tabular}




\begin{tabular}{|c|c|c|}
\hline Avaliação da estrutura do curso & n & $(\%)$ \\
\hline Concordo mais ou menos & 19 & $(16,2)$ \\
\hline Concordo & 62 & $(53,0)$ \\
\hline Concordo totalmente & 32 & $(27,3)$ \\
\hline \multicolumn{3}{|c|}{ O material do curso foi organizado de forma lógica e compreensível } \\
\hline Discordo totalmente & 0 & $(0)$ \\
\hline Discordo & 1 & $(0,9)$ \\
\hline Concordo mais ou menos & 13 & $(11,1)$ \\
\hline Concordo & 66 & $(56,4)$ \\
\hline Concordo totalmente & 37 & $(31,6)$ \\
\hline \multicolumn{3}{|c|}{$\begin{array}{l}\text { O curso foi organizado de forma adequada para aprender os conceitos } \\
\text { descritos nos objetivos do curso }\end{array}$} \\
\hline Discordo totalmente & 1 & $(0,9)$ \\
\hline Discordo & 1 & $(0,9)$ \\
\hline Concordo mais ou menos & 18 & $(15,3)$ \\
\hline Concordo & 68 & $(58,1)$ \\
\hline Concordo totalmente & 29 & $(24,8)$ \\
\hline \multicolumn{3}{|l|}{ Este curso online foi fácil de usar } \\
\hline Discordo totalmente & 1 & $(0,9)$ \\
\hline Discordo & 0 & $(0)$ \\
\hline Concordo mais ou menos & 12 & $(10,3)$ \\
\hline Concordo & 63 & $(53,8)$ \\
\hline Concordo totalmente & 41 & $(35,0)$ \\
\hline \multicolumn{3}{|l|}{ As páginas foram claramente definidas } \\
\hline Discordo totalmente & 0 & $(0)$ \\
\hline Discordo & 2 & $(1,7)$ \\
\hline Concordo mais ou menos & 12 & $(10,3)$ \\
\hline Concordo & 65 & $(56,0)$ \\
\hline Concordo totalmente & 37 & $(32,0)$ \\
\hline
\end{tabular}

Fonte: dos autores.

A Tabela 3 apresenta os percentuais dos escores de avaliação de satisfação dos pós-graduandos com o curso. Sobre o questionamento se a qualidade acadêmica foi parecida com cursos presenciais dos quais os participantes já fizeram parte, observouse avaliações positivas em $63,3 \%$ da amostra e $23 \%$ relataram que concordam mais ou menos. A maioria, com resposta concorda e concorda totalmente $(81,2 \%)$, relatou que gostaria de participar de outra oferta de cursos deste tipo e $83,8 \%$ recomendaria o curso para outras pessoas. 
Tabela 3 - Avaliação da satisfação de pós-graduandos matriculados em um curso de especialização na modalidade EaD utilizando uma escala de likert

\begin{tabular}{lccc}
\hline & Avaliação de satisfação & n & (\%) \\
\hline A qualidade acadêmica foi parecida com cursos presenciais que já fiz & & \\
\hline Discordo totalmente & 4 & $(3,4)$ \\
Discordo & 12 & $(10,3)$ \\
Concordo mais ou menos & 27 & $(23,0)$ \\
Concordo & 62 & $(53,0)$ \\
Concordo totalmente & 12 & $(10,3)$ \\
\hline Gostaria de participar de outra oferta de cursos deste tipo & & \\
\hline Discordo totalmente & 3 & $(2,6)$ \\
Discordo & 6 & $(5,1)$ \\
Concordo mais ou menos & 13 & $(11,1)$ \\
Concordo & 65 & $(55,6)$ \\
Concordo totalmente & 30 & $(25,6)$ \\
\hline Eu recomendaria este curso para outras pessoas & & \\
\hline Discordo totalmente & 2 & $(1,7)$ \\
Discordo & 4 & $(3,4)$ \\
Concordo mais ou menos & 13 & $(11,1)$ \\
Concordo & 66 & $(56,4)$ \\
Concordo totalmente & 32 & $(27,4)$ \\
\hline
\end{tabular}

Fonte: dos autores.

A distribuição da avaliação de aprendizagem está descrita na Tabela 4. Dentre as variáveis analisadas, os questionamentos sobre aprendizagem foram os que apresentaram percentuais mais baixos de avaliações positivas. Sobre a comparação entre curso online e curso presencial, 49,6\% concordam ou concordam totalmente na igualdade no aprendizado, $30,5 \%$ concordam ou concordam totalmente que aprenderam mais em cursos online do que em cursos presenciais, e 35\% concordam ou concordam totalmente que a qualidade da experiência de aprendizagem em cursos online é melhor do que em cursos presenciais.

Tabela 4 - Avaliação do resultado de aprendizagem de pós-graduandos matriculados em um curso de especialização na modalidade $\mathrm{EaD}$ utilizando uma escala de likert

\begin{tabular}{lccc}
\hline \multicolumn{1}{c}{ Avaliação do resultado de aprendizagem } & n & (\%) \\
\hline $\begin{array}{l}\text { Eu sinto que eu aprendi tanto com este curso como eu poderia ter } \\
\text { aprendido num curso presencial }\end{array}$ & & \\
\hline Discordo totalmente & 4 & $(3,4)$ \\
Discordo & 25 & $(21,4)$ \\
Concordo mais ou menos & 30 & $(25,6)$
\end{tabular}




\begin{tabular}{lcc}
\hline \multicolumn{1}{c}{ Avaliação do resultado de aprendizagem } & n & (\%) \\
\hline Concordo & 37 & $(31,6)$ \\
Concordo totalmente & 21 & $(18,0)$ \\
\hline Eu sinto que eu aprendi mais em cursos online do que em cursos & & \\
presenciais & & \\
\hline Discordo totalmente & 11 & $(9,3)$ \\
Discordo & 36 & $(30,5)$ \\
Concordo mais ou menos & 35 & $(29,7)$ \\
Concordo & 25 & $(21,2)$ \\
Concordo totalmente & 11 & $(9,3)$ \\
\hline A qualidade da experiência de aprendizagem em cursos online é melhor & & \\
do que em cursos presenciais & & \\
\hline Discordo totalmente & 10 & $(8,6)$ \\
Discordo & 36 & $(30,8)$ \\
Concordo mais ou menos & 30 & $(25,6)$ \\
Concordo & 30 & $(25,6)$ \\
Concordo totalmente & 11 & $(9,4)$ \\
\hline
\end{tabular}

Fonte: dos autores.

A Tabela 5 descreve as medidas de correlação entre as dimensões de avaliação do curso. Observou-se que todas as dimensões apresentaram correlações diretamente proporcionais e estatisticamente significantes. As dimensões que apresentaram medidas de correlação positivas mais fortes foram: satisfação e aprendizagem $(\mathrm{Rs}=0,714 ; \mathrm{P}<$ 0,001), satisfação e conteúdo ( $\mathrm{Rs}=0,658 ; \mathrm{P}<0,001)$.

Tabela 5 - Análise de correlação entre as dimensões de avaliação do curso

\begin{tabular}{lccc}
\hline Correlações & $\begin{array}{c}\text { Avaliação da } \\
\text { Estrutura do Curso }\end{array}$ & $\begin{array}{c}\text { Avaliação de } \\
\text { Satisfação com o } \\
\text { Curso }\end{array}$ & $\begin{array}{c}\text { Avaliação de } \\
\text { Aprendizagem no } \\
\text { Curso }\end{array}$ \\
\hline \multirow{2}{*}{ Avaliação de Conteúdo } & $\mathrm{Rs}=0,545$ & $\mathrm{Rs}=0,658$ & $\mathrm{Rs}=0,476$ \\
& $\mathrm{P}<0,001^{*}$ & $\mathrm{P}<0,001^{*}$ & $\mathrm{P}<0,001^{*}$ \\
\hline \multirow{2}{*}{ Avaliação da Estrutura } & - & $\mathrm{Rs}=0,560$ & $\mathrm{Rs}=0,369$ \\
& - & $\mathrm{P}<0,001^{*}$ & $\mathrm{P}<0,001^{*}$ \\
\hline \multirow{2}{*}{ Avaliação de Satisfação } & - & - & $\mathrm{Rs}=0,714$ \\
\end{tabular}

Legenda: $\mathrm{Rs}=$ Coeficiente de Spearman. $\mathrm{P}=$ valor de probabilidade. ${ }^{*}$ Estatisticamente significante $(\mathrm{P}<0,05)$.

Fonte: dos autores. 


\section{DISCUSSÃO}

A UNA-SUS/UFMA, sendo uma instituição de ensino que trabalha com a modalidade $\mathrm{EaD}$, possui como objetivos: disponibilizar aos trabalhadores da saúde a oferta de cursos adequados à realidade local, utilizando-se de interações presenciais e a distância, visando a capacitação em áreas estratégicas para o SUS; criar um acervo público e colaborativo de materiais educacionais para área da saúde; promover a incorporação de novas Tecnologias de Informação e Comunicação aos processos de Educação em Saúde e, por esse motivo, preocupa-se em sempre aprimorar seu material educacional. Logo, torna-se crucial conhecer e entender cada vez mais o seu público.

Com base nos resultados desta pesquisa, pode-se concluir que, em relação aos escores de avaliação do conteúdo do curso ofertado pela instituição, todas as questões desse bloco tiveram avaliações positivas, acima de 70\%, o que indica um alto grau de satisfação em relação ao conteúdo disponibilizado, sendo dentre as medidas de correlação entre as dimensões do curso uma das mais altas (Tabela 5). Desse modo, infere-se que o conteúdo ofertado é de grande valia para o aluno, sendo aplicável em sua prática, bem como os materiais e recursos do curso melhoraram a aprendizagem, tornando-a mais eficiente dentro do contexto de EPS, ao levar em consideração a prática profissional. Segundo Puerta e Amaral (2012), para o aluno, a partir do momento que a EaD apresenta possibilidades concretas para o aprendizado (transferência da teoria para a prática), há uma transformação da realidade, agregando e criando valores para esses alunos. A aprendizagem colaborativa é importante para o compartilhamento de um objetivo em comum e, através de uma metodologia interativa, há a promoção de uma relação afetiva, reflexiva e autônoma com o conhecimento (VIANNA ATAIDE; FERREIRA, 2015).

Sobre os resultados relacionados e estrutura do curso, todos os questionamentos obtiveram avaliações positivas. A estruturação do curso implica diretamente no processo de aprendizagem do usuário, pois faz parte do processo de comunicação entre alunoconteúdo. $\mathrm{O}$ curso aqui avaliado provou-se fácil de usar, de acordo com a avaliação dos alunos, bem estruturado e bem definido, segundo os resultados, além de contemplar as diretrizes citadas.

Quanto às questões que comparavam cursos online e cursos presenciais no quesito equivalência na aprendizagem, muitos alunos confirmaram ter aprendido num curso online tanto quanto em um presencial. Este dado pode indicar uma percepção positiva dos alunos quanto à aprendizagem online, o que se justificaria pelo dinamismo da modalidade, pela interatividade e pelos recursos educacionais oferecidos. Além disso, os alunos gostariam de participar novamente de uma oferta de cursos na modalidade $\mathrm{EaD}$, bem como indicariam o curso para outras pessoas.

Por outro lado, quando se trata de $\mathrm{EaD}$ é preciso que algumas questões sejam consideradas, como a eficácia de alguns recursos tecnológicos, tais como e-books. Por vezes, este recurso pode estar organizado de maneira complexa, dificultando a localização de determinados conteúdos dentro do material e o trajeto dos alunos 
para refazerem os caminhos até as informações desejadas. Ademais, durante a leitura de livros digitais, os estudantes podem navegar entre diversas mídias digitais que competem por sua atenção, o que pode gerar uma série de distrações online, segundo Falc (2013).

Para otimizar a utilização dos materiais digitais, é interessante que se possibilite a impressão dos mesmos (o que a UNA-SUS/UFMA já faz ao disponibilizar o material em PDF), bem como faz-se necessária a inserção de alguns recursos nestes materiais, por exemplo, permitir que o aluno possa marcar partes importantes ou copiá-las, de maneira que se possibilite guardar essas informações também de forma permanente.

Comparando-se as modalidades presencial e à distância, a segunda permite maior flexibilidade em relação ao tempo para leitura e reflexão do material, deixando o aluno livre para organizar sua rotina, permitindo uma maior disponibilidade de tempo e ritmos de estudos diferenciados, além de ser mais dinâmica e possuir diversos recursos educacionais, diferentemente do modelo presencial, onde esse tempo é mais restrito e há dificuldade para reunir o grupo para desenvolvimento do trabalho final da disciplina. Além disso, a $\mathrm{EaD}$ pode gerar ofertas tanto para uma única pessoa quanto para grupos, o que permite uma maior abrangência de pessoas que podem acessar o curso. Há também uma democratização do ensino, já que engloba uma diversidade cultural, social e econômica (SILVA; SILVA, 2012).

A EaD também proporciona a utilização de recursos dentro da própria instituição de trabalho, o que é uma motivação para aprender com o uso da tecnologia e a possibilidade de não ter que sair do local de trabalho para participar de um curso (GODOY, 2004, apud SILVA et al., 2015).

Considerando que cada modalidade de ensino é diferente e apresenta suas particularidades, vantagens e desvantagens, a conciliação entre as duas metodologias de ensino pode ser considerada uma solução para diversos problemas, combinandoas quando necessário para diminuir as lacunas entre elas. Ao se escolher a maneira mais adequada de ensino e apresentação do conteúdo, contribui-se de forma positiva e significativa para o processo de aprendizado (GARDNER, 1995, apud SANTOS; BOTTENTUIT JUNIOR, 2015).

\section{CONCLUSÃO}

A presente pesquisa tornou possível conhecer o nível de satisfação dos alunos de um Curso de Especialização, realizado na modalidade $\mathrm{EaD}$ no que diz respeito ao conteúdo ofertado, à estrutura, bem como à aprendizagem obtida.

Esse processo mostra a importância de reconhecimento do aluno como parte do processo de aperfeiçoamento da aprendizagem, principalmente na modalidade a distância e no processo da Educação Permanente em Saúde, onde os elementos do curso ofertado, sua estrutura, conteúdo, recursos e a própria aprendizagem em si devem 
ser planejados, organizados, estruturados e produzidos de maneira a contemplar o principal objetivo, que é a possibilidade de aprendizado, com a transferencia da teoria para a prática, levando em conta a satisfação do aluno.

\section{REFERÊNCIAS}

BONICI, Rosangêla Maura Correia; ARAÚJOJÚNIOR, Carlos Fernando de. Medindo a satisfação dos estudantes em relação a disciplina on-line de Probabilidade e Estatística. In: CONGRESSO INTERNACIONAL DE EDUCAÇÃO A DISTÂNCIA, 17., 2011, Manaus, AM, 2011. Disponível em: 〈http://www.abed.org.br/congresso2011/trabalhos1.asp〉. Acesso em: 17 ago. 2015.

CENSO EaD.Br. Associação Brasileira de Educação a Distância - ABED (Org.). Relatório analítico da aprendizagem a distância no Brasil 2013. 1 ed. Curtitiba: Ibpex, 2014. 332p. ISBN: 978-85-417-0055-9. Disponível em: <http://www.abed.org.br/censoead2013/CENSO_ EAD_2013_PORTUGUES.pdf $>$. Acesso em: 22 out. 2015.

FALC, Emilie O. An Assessment of College Students' Attitudes towards using an online e- textbook. Interdisciplinary Journal of E-Learning and Learning Objects, Minnesota, EUA, v. 9, 2013. Disponível em: <http://www.ijello.org/Volume9/IJELLOv9p001012Falc831.pdf >. Acesso em: 17 ago. 2015.

GARCIA, Paola Trindade et al. Proposta de construção de design instrucional: concepção, elaboração e aspectos para produção de recursos multimídia da UNA-SUS/ UFMA. In: CONGRESSO NACIONAL DE AMBIENTES HIPERMÍDIA PARA APRENDIZAGEM CONAHPA, 7., 2015, Maranhão, 2015.

MAYADAS, A. Frank; BOURNE, John; BACSICH, Paul. Online education today. Science, v. 323, n. 5910, p. 85-89, jan. 2009. Disponível em: <http://www.sciencemag.org/ content/323/5910/85>. Acesso em: 17 ago. 2015.

MOORE, Michael G.; KEARSLEY, Greg. Educação a distância: uma visão integrada. Tradução de Roberto Galman. São Paulo: Thomson Learning, 2007.

NOGUEIRA, Luís Lindolfo. Educação a distância. Comunicação e Educação, São Paulo, v. 5, p. 34 - 39, jan./abr.1996.

OLIVEIRA, Ana Emilia Figueiredo de et al. Aplicativo aberto para dispositivos móveis e sua aplicabilidade na atenção básica: a experiência da UNA-SUS/UFMA. 2014. Disponível em: <http://www.unasus.gov.br/sites/default/files/livro_relatos_una-sus_2014.pdf>. Acesso em: 17 jun. 2015.

PACIEVITCH, Thais. Tecnologia da informação e comunicação. InfoEscola, 2015.

Disponível em: <http://www.infoescola.com/informatica/tecnologia-da-informacao-ecomunicacao/.> Acesso em: 17 maio. 2015.

PUERTA, Adriana Amaral; AMARAL, Roniberto Morato do. Comparação da educação presencial com a educação à distância através de uma pesquisa aplicada. In: SEMINÁRIO NACIONAL DE BIBLIOTECAS UNIVERSITÁRIAS - SNBU, 15., 2012. São Paulo, 
2012. Disponível em: <http://www.sbu.unicamp.br/snbu2008/anais/site/pdfs/2866.pdf>. Acesso em: 17 maio. 2015.

SANTOS, Thays Fernanda Silva dos; BOTTENTUIT JUNIOR, João Batista. Perspectivas da Teoria das Inteligências Múltiplas para a Educação a Distância: um estudo no curso de licenciatura em pedagogia do NEAD-UFMA. Revista EducaOline, Rio de Janeiro, v. 9, n. 1, jan./abr. 2015. Disponível em: <http://www.latec.ufrj.br/revistas/index.php?journal=educao nline\&page $=$ article\&op $=$ view\&path\%5B\%5D =639\&path\%5B $\% 5 \mathrm{D}=655>$.

SCHROEDER, Christine da Silva. Educação a distância e mudança organizacional na Escola de Administração da UFRGS: uma teoria substantiva. 2009. 252f. Tese (Doutorado em Administração) - Programa de Pós-Graduação em Administração, Universidade Federal do Rio Grande do Sul, Porto Alegre, 2009. Disponível em: <http://www.ufrgs.br/gpnavi/ artigos/Tese_Christine.pdf >. Acesso em: 18 ago. 2015.

SILVA, Adriane das Neves Silva et al. Limites e possibilidades do ensino à distância (EaD) na educação permanente em saúde: revisão integrativa. Ciência \& Saúde Coletiva, v. 20, n. 4, p. 1099-1107, 2015. Disponível em: <http://www.scielosp.org/pdf/csc/v20n4/1413-8123csc-20-04-01099.pdf>. Acesso em: 22 set. 2015.

SILVA, André Luiz; SILVA, Priscila Maíla da. Educação superior à distância: uma alternativa de acesso ao saber acadêmico através do ciberespaço. Revista Temática, Paraíba, ano. 8, n. 9, nov. 2012. Disponível em: <http://periodicos.ufpb.br/ojs2/index.php/tematica/ article/view/23159/12738>. Acesso em 22 set. 2015.

VIANNA, Luciano José; ATAIDE, Cintia Aparecida; FERREIRA, Marussa Campos. Educação a distância no Brasil: cotidiano, prática, avanços e perspectivas. In: ENCONTRO INTERNACIONAL DE FORMAÇÃO DE PROFESSORES, 8., FÓRUM PERMANENTE DE INOVAÇÃO EDUCACIONAL, 9., 2015. Anais... Aracaju: Unit, 2015. Disponível em: <https://eventos.set.edu.br/index.php/enfope/article/view/1635/176>. Acesso em: 22 set. 2015. 